\title{
A Survey on Energy Efficient Clustering Protocols in Wireless Sensor Networks
}

\author{
Nazia Farooq \\ Department of IT \\ Central University of Kashmir
}

\author{
M. Abdul Jawad \\ Department of IT \\ Central University of Kashmir
}

\begin{abstract}
A Wireless Sensor Network is a network of randomly deployed sensor nodes that cooperatively monitor the physical and environmental conditions. Such networks face some critical issues like limited battery power, limited network lifetime and deployment in drastic environmental conditions. The performance of Wireless Sensor Network largely depends on the routing protocols. The lifetime of the sensor network primarily depends on the residual battery of each sensor node. Many routing protocols have been proposed to optimize the energy efficiency of the Sensor Networks. We have carried out an extensive survey on WSN routing protocols. Clustering routing protocols out perform all other routing protocols in terms of extending the network lifetime. This paper mainly focuses on an in-depth survey of hierarchical or clustering routing protocols like LEACH and PEGASIS and their descendants.
\end{abstract}

\section{Keywords}

Sink, LEACH, PEGASIS, Network Lifetime, Clustering

\section{INTRODUCTION}

The recent technological advances in micro-electronics have made it possible to manufacture small and low cost sensors that are both technically and economically feasible. The progression of wireless sensor networks was initially motivated by military applications but in current times their applications have scaled from environment monitoring, object tracking, acoustic and seismic detection, traffic control automation, healthcare to their roles in making internet of things (IOT) practical. A Wireless Sensor Network (WSN) is an internetwork of hundreds or even thousands of tiny nodes known as Sensors. These nodes communicate using multi-hop routing and eventually transmit the sensed data to a special node known as Sink Node or Base Station (BS). The Sensor nodes are usually deployed in a remote area of interest where they operate in harsh or extreme environmental conditions. These sensor nodes coordinate among themselves to disseminate the information sensed about a particular phenomenon, given their ability to both sense and transmit the data to base station either directly or using multiple hops. The base station can be either fixed or mobile in wireless sensor network and in some cases the network may consist of more than one base station. The layout design of sensors in an area is completely application specific and environment dependent. However the node deployment strategy has a serious impact on the performance of the wireless sensor network. Ever since the evolution of wireless sensor networks, energy conservation has been a major challenge and it has become an area of focus in both academia and industry. This energy can be very expensive, difficult and even impossible to renew. So network lifetime enhancement in wireless sensor networks is a critical issue.

The communication and component architecture of wireless sensor network is shown in fig. 1. The essential components of a sensor node are: a micro sensor, a microcontroller, a transceiver and a battery. In addition the mobilizer and position finding system are optional and are application specific.

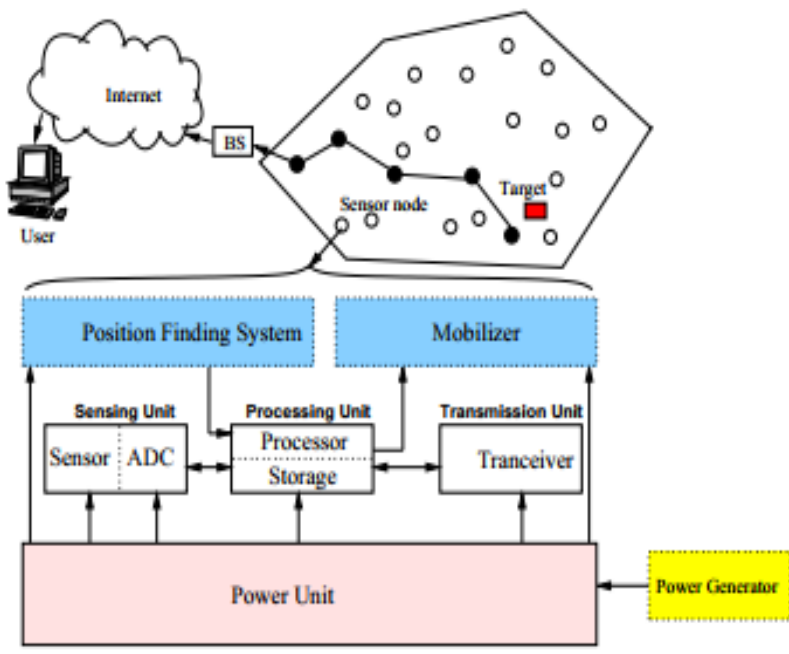

Figure 1 Communication and Component Architecture of WSN

In Wireless sensor networks nodes mostly deplete their energy in processing and in communicating (transmitting and receiving) with adjacent nodes. The energy dissipation is in accordance with the first order radio model. The general mode of communication used in wireless sensor networks is radio communication and it is impossible with such communication facility for every sensor node to reach the Base Station directly. To meet the constraint, hop-by-hop data transfer is used by nodes to deliver data to destination. The hop-by-hop communication increases the overhead on the sensor nodes that are close to base station as all transmission take place through these nodes only (Hotspot Effect). In addition, a lot of energy is consumed in various states that are useless from application point of view like idle listening, overhearing, interference and collision.

Routing protocols discover and maintain energy efficient routes, so as to make communication efficient and reliable. On the basis of network structure WSN routing protocols are divided into three categories: flat routing, hierarchical routing (cluster based) and location based routing. In flat networks every node is assigned the same role and similar capabilities. The individual sensor nodes cooperatively work to perform the desired sensing task and finally transmitting to the base station or sink. This model works for small scale networks but as the deployment is increased uneven energy depletion and reduced network lifetime is observed. Hierarchical networks support heterogeneous sensing and nodes are typically grouped into clusters having some common requirements. 
Clustering involves creation of clusters and cluster heads $(\mathrm{CHs})$ that significantly improve the network lifetime and achieve balanced energy dissipation in wireless sensor networks. Clustering uses aggregation of data to reduce redundancy and communication load caused by multiple adjacent nodes, then sending the aggregated data to the next Cluster Head or Base Station where it is processed, stored and retrieved.

\section{LITERATURE REVIEW}

The traditional routing protocols aren't optimal in wireless sensor networks when it comes to energy consumption. Hierarchical routing protocols provide a balanced and efficient energy consumption. They do this by dividing nodes into different clusters. In each cluster a powerful node (higher energy node) known as Cluster Head is used to process and transmit energy to the Base Station while low energy nodes are mainly assigned the tasks of sensing. Fig. 2 shows a hierarchical cluster that is divided into different clusters. The cluster consists of sensor nodes and a Cluster Head that takes responsibility of sending data from cluster to other Cluster Heads or Base Station. In hierarchical model the cluster head performs the data aggregation process, while in multi-hop routing each intermediate node performs the data aggregation and data forwarding. The architecture allows reduced number of transmissions performed for disseminating the routing information to Base Station or Sink. In Wireless Sensor Networks, clustering faces several deployment issues Cluster Formation, Ensuring Connectivity, Cluster Head Selection, Real-Time Operation, Synchronization, Data Aggregation and Quality of Service.

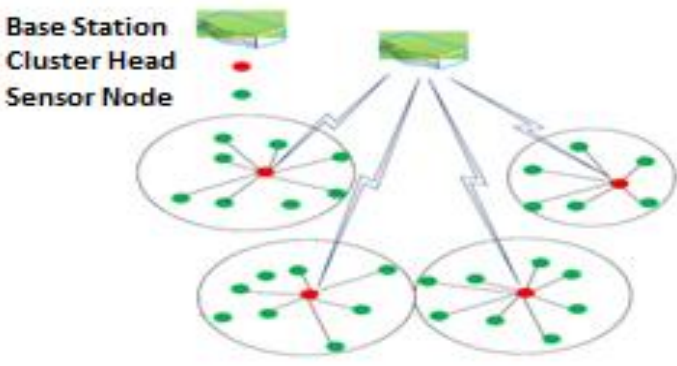

Figure 2 Hierarchical Clustering Model

Heinzelman et. al. [1] proposed a hierarchical clustering algorithm - LEACH (Low Energy Adaptive Clustering Hierarchy) for routing in distributed homogenous networks. LEACH initially selects the cluster heads randomly and the role changes to different nodes according to round- robin management policy to ensure fair energy dissipation between nodes. The cluster head compresses the data arriving from the member nodes of the cluster and send an aggregated packet to the base station to reduce the number of transmissions to the destination. LEACH uses TDMA/CDMA MAC to reduce inter-cluster and intra-cluster collisions. LEACH operates in two phases - setup phase and steady phase.

In Setup Phase, Cluster Heads are selected and clusters are formed while the Steady Phase takes the responsibility of data transfer to base station from individual clusters. The First Phase starts with Cluster Formation and assigning Cluster Heads. Initially, the sensor nodes choose a random number ' $\mathrm{m}$ ' between 0 and 1 . If this number ' $\mathrm{m}$ ' is less than the threshold value $T(n)$, then sensor node becomes the cluster head for the current round. The value of $T(n)$ is calculated based on the equation that includes the fraction of value to become a cluster head, the current round and the set of nodes that have not been selected as cluster-head in the last $(1 / p)$ rounds denoted by $\mathrm{G}$.

$$
T(n)=\frac{p}{1-p(r \bmod (1 / p))} \quad \text { if } n \in G
$$

Where $\mathrm{G}$ is the set of nodes that are involved in the Cluster Head election. The elected Cluster Head broadcasts an advertisement packet to rest of the network that it is the new cluster head. The sensor nodes after receiving the advertisement decide onto which cluster they want to belong to. The decision is purely based on signal strength of the advertisement. The cluster head creates a TDMA schedule and assigns each node a time slot when it can transmit. The steady state starts with data transmission where individual nodes sense data and send this sensed data to their respective cluster head. LEACH is a power clustering routing protocol that increases overall network lifetime by rotating cluster head roles. LEACH assumes that all nodes can transmit data with enough power to reach the destination. So LEACH isn't suitable for networks with high coverage areas. Since cluster heads are elected randomly, low energy nodes can also be elected as cluster heads even when high energy nodes are available. Failure of cluster head leads to lack of robustness. The data aggregation in LEACH is centralized and is performed periodically. However, sometimes the periodic transmission of data may not be necessary, otherwise exhausting the energy levels of nodes very rapidly.

LEACH-C [2] contrary to LEACH is a centralized clustering approach that exhibits data transmission which is dependent on location awareness and energy levels of each sensor node. Sensor nodes with energy levels greater than the minimum defined threshold are eligible for Cluster. In LEACH-C since the cluster head is elected by base station itself it is guaranteed that desired number of cluster heads will be created and evenly distributed among nodes. However, LEACH-C is sensitive to the sink location. Once the energy cost of communicating with the sink becomes higher than the energy cost for cluster formation, LEACH-C no longer provides good performance. Sinks may be located far from the network in most WSN applications. So, the dependence on the sink location is a major disadvantage of LEACH-C.

LEACH-E [3] (Energy Low Energy Adaptive Clustering Hierarchy) protocol is an improvement over LEACH. Initially the cluster head is selected randomly and each node has equal probability of being selected as cluster head. In the subsequent rounds the residual energy of nodes determine which sensor node will become the cluster head. This ensures that low energy nodes are only used for sensing and thereby overall network lifetime of network gets increased.

LEACH-F [4] (Fixed number of Cluster Low energy Adaptive Clustering Hierarchy). LEACH-F avoids re-clustering uses a stable cluster and rotating cluster head in which cluster head once formed is maintained throughout. Initially the clusters are formed and cluster heads are formed using LEACH-C algorithm. The major limitation of this protocol is clusters once formed are permanent and new nodes cannot be added to the clusters where nodes die because of energy drainage.

Modified LEACH (MODLEACH) [5] eliminates the need of unnecessary routing overheads because of cluster head replacement in each new round. The scheme incorporates dual transmission power for sensor nodes allowing farther and nearer nodes from the base station to transmit at different power levels. 
Table 1. Comparison of LEACH Descendent Protocols

\begin{tabular}{|l|l|l|l|}
\hline $\begin{array}{l}\text { LEACH } \\
\text { Descendent }\end{array}$ & $\begin{array}{l}\text { Clustering } \\
\text { Method }\end{array}$ & $\begin{array}{l}\text { Data } \\
\text { Aggregation }\end{array}$ & Scalability \\
\hline LEACH & Distributed & YES & Limited \\
\hline LEACH-C & Centralized & YES & Good \\
\hline LEACH-E & Distributed & YES & Very Good \\
\hline LEACH-F & Centralized & YES & Limited \\
\hline MODLEACH & Distributed & YES & Good \\
& & & \\
\end{tabular}

Power Efficient Gathering in Sensor Information Systems (PEGASIS) [6] is an improvement over LEACH and provides optimal data gathering in sensor networks. The protocol works by establishing a chain among sensor nodes so that each node will receive and transmit to a close neighbor. The aggregated data moves from node to node and eventually reaches to the base station. Nodes take turns in transmitting to the base station so that average energy spent by each node per round is reduced. PEGASIS is based on pre-assumption that each node has the global knowledge of the network. Every node limits its signal strength so that it is in range of a single adjacent node. The chain thus formed is of nodes that are closest to each other and form a path to base station. Simulation results prove that PEAGSIS is able to extend the network lifetime by a factor of 2 when compared to LEACH. Moreover PEGASIS assumes that each sensor node has the ability to directly communicate to base station and all sensor nodes have same level of energy and are likely to die at the same time. PEGASIS isn't a feasible option for networks with dynamic or varying topology. As the size of network will be larger, the delay in transmission will be long. However a single leader can become a bottleneck.

Khamforoosh et. al. proposed PEGASIS for energy reduction where chain structure is like it always gives the minimum distance to the destination thereby reducing the total energy consumption [7]. A study by Sen, Feng, Qi Bing, and Tang Liangrui improved the PEGASIS as Energy Efficient PEGASIS based protocol (EEPB) [8]. The protocol selects the leader by considering both residual energy of nodes and distance between node and base station. Another study by Jafri, Mohsin Raza, Nadeem Javaid and et. al. implemented improved energy efficient PEGASIS based routing protocol with sink mobility (MIEEPB). Since the sink is mobile, smaller chains and reduced load on Leaders was observed. Moreover mobile sink minimizes the energy used by sensor nodes and reduces the data delivery delay for nodes in the network.

Table 2. Comparison of PEGASIS Descendent Protocols

\begin{tabular}{|l|l|l|}
\hline Protocol & Scalability & $\begin{array}{l}\text { Energy } \\
\text { Efficiency }\end{array}$ \\
\hline PEGASIS & Good & Good \\
\hline EEPB & Good & High \\
\hline MIEEPB & High & Very High \\
\hline
\end{tabular}

\section{CONCLUSION}

Wireless Sensor Networks are deployed over large geographical area and they find applications in many areas. Battery Power or Residual Energy is a major concern in such networks. Various protocols and approaches addressed this issue in wireless sensor networks. This Paper presented a detailed survey of clustering protocols that somehow largely address this problem. The work focused on highlighting the merits and demerits of different cluster based routing protocols. Among the clustering based routing protocols PEGASIS and its descendants outperform other routing protocols in terms of extending the network lifetime and quality of network in wireless sensor networks.

\section{REFERENCES}

[1] W. Heinzelman, A. Chandrakasan and H. Balakrishnan, "Energy-E-cient Communication Protocol for Wireless Microsensor Networks," Proceedings of the 33rd Hawaii International Conference on System Sciences (HICSS '00), January 2000.

[2] Heinzelman.W,Chandrakasan.A, Balakrishnan.H: An application-specific protocol architecture for wireless micro sensor networks: IEEE Transaction on Wireless Communications, Vol. 1, No. 4, pp. 660-670, 2002.

[3] Fan. X. N, Song. Y. L: Improvement on LEACH protocol of wireless sensor network. In: Proc. International Conference on Sensor Technologies and Applications, Sensor Comm., pp. 260-264, 2007.

[4] Manimala.P, Senthamil.R: A Survey on Leach-Energy Based Routing Protocol. International Journal of Emerging Technology and Advanced Engineering (IJETAE), Vol.3, Issue 12, pp. 657-660, December, 2013.

[5] Khamforoosh, Keyhan, and Hana Khamforoush. A New Algorithm for Energy Reduction in Wireless Sensor Networks.2nd IEEE International Conference on Computer Science and Information Technology 2009; $505-509$.

[6] S. Lindsey, C. Raghavendra, PEGASIS: Power-E-cient Gathering in Sensor Information Systems", IEEE Aerospace

Conference Proceedings, 2002, Vol. 3, 9-16 pp. 11251130.

[7] Khamforoosh, Keyhan, and Hana Khamforoush. A New Algorithm for Energy Reduction in Wireless Sensor Networks.2nd IEEE International Conference on Computer Science and Information Technology 2009;505-509.

[8] Sen, Feng, Qi Bing, and Tang Liangrui. An Improved Energy-Efficient PEGASIS-Based Protocol in Wireless Sensor Networks. Fuzzy Systems and Knowledge Discovery (FSKD), 2011 Eighth International Conference On. Vol. 4. IEEE, 2011.

[9] Jafri, Mohsin Raza, Nadeem Javaid, Akmal Javaid, and Zahoor Ali Khan. Maximizing the lifetime of multichain pegasis using sink mobility. World Applied Sciences Journal, vol.21, no.9, March 2013; 1283-1289.

[10] W. Heinzelman, J. Kulik, and H. Balakrishnan, "Adaptive Protocols for Information Dissemination in Wireless Sensor Networks," Proc. 5th ACM/IEEE Mobicom Conference (MobiCom '99), Seattle, WA, August, 1999. pp. 174-85.

[11] I. Akyildiz, W. Su, Y. Sankarasubramaniam, and E. Cayirci, "A survey on sensor networks," IEEE Communications Magazine, Volume: 40 Issue: 8, pp.102-114, August 2002. 
[12] A. Perrig, R. Szewzyk, J.D. Tygar, V. Wen, and D. E. Culler, "SPINS: security protocols for sensor networks". Wireless Networks Volume: 8, pp. 521-534, 2000.

[13] S. Hedetniemi and A. Liestman, \A survey of gossiping and broadcasting in communication networks", IEEE Networks, Vol. 18, No. 4, pp. 319-349, 1988.

[14] J. Kulik, W. R. Heinzelman, and H. Balakrishnan, "Negotiation-based protocols for disseminating information in wireless sensor networks," Wireless Networks, Volume: 8, pp. 169-185, 2002.
[15] A. Manjeshwar and D. P. Agarwal, "TEEN: a routing protocol for enhanced e-ciency in wireless sensor networks," In 1st International Workshop on Parallel and Distributed Computing Issues in Wireless Networks and Mobile Computing, April 2001.

[16] A. Manjeshwar and D. P. Agarwal, "APTEEN: A hybrid protocol for efficient routing and comprehensive information retrieval in wireless sensor networks," Parallel and Distributed Processing Symposium., Proceedings International, IPDPS 2002, pp. 195-202. 